\title{
SÍNDROME METABÓLICA E FATORES DE RISCO CARDIOVASCULAR EM POLICIAIS MILITARES: UMA REVISÃO DA LITERATURA ${ }^{1}$
}

\author{
METABOLIC SYNDROME AND CARDIOVASCULAR \\ RISK FACTORS IN MILITARY POLICE: A LITERATURE REVIEW
}

\author{
Andressa Lorenzoni Righi², Cristiana Basso ${ }^{3}$ e Natielen Jacques Schuch ${ }^{4}$
}

\section{RESUMO}

Este trabalho teve como objetivo investigar a ocorrência de Síndrome Metabólica (SM) e Fatores de Risco Cardiovascular em Policiais Militares. Tratou-se de uma revisão bibliográfica integrativa realizada nas bases de dados LILACS, PubMed e SciELO. Foram selecionados artigos do período de 2010 a 2020, disponíveis na íntegra, nas línguas portuguesa, inglesa e espanhola, sendo excluídos os que se enquadravam fora do recorte temporal, duplicatas na mesma base de pesquisa, teses, dissertações, artigos de revisão, protocolos e recomendações. Foram identificados 622 artigos nas bases de dados referenciadas, selecionando-se, após refinamentos, 11 estudos, sendo 6 na plataforma PubMed, 3 na biblioteca SciELO e 2 na base Lilacs. Observaram-se índices consideráveis de sobrepeso e obesidade, além de risco cardiovascular muito elevado de acordo com a circunferência da cintura/abdominal e relação cintura/quadril. Constataram-se ainda, níveis alterados de glicemia em jejum e triglicerídeos, além de baixos níveis de colesterol de lipoproteína de alta densidade. Os estudos elegidos apontaram a frequente ocorrência de fatores de risco cardiovascular em policiais militares, com destaque para elevados índices de excesso de peso corporal. Ademais, verificou-se a existência de dados de prevalência de SM relativamente expressivos nas forças policiais, fato que suscita atenção especial demandando a realização de novos estudos acerca do tema.

Palavras-chave: Antropometria, Doenças cardiovasculares, Obesidade, Policial.

\section{ABSTRACT}

This study aimed to investigate the occurrence of Metabolic Syndrome and Cardiovascular Risk factors in Military Police. It was an integrative bibliographic review carried out in the LILACS, PubMed and SciELO databases. Articles from the period 2010 to 2020 were selected, fully available, in the Portuguese, English and Spanish languages, excluding those that fell outside the time frame, duplicates in the same research base, theses, dissertations, review articles, protocols and recommendations. 622 articles were identified in the referenced databases, selecting, after refinements, 11 studies, 6 in the PubMed platform, 3 in the SciELO library and 2 in the Lilacs database. Considerable rates of overweight and obesity were observed, in addition to very high cardiovascular risk according to waist / abdominal circumference and waist / hip ratio. Altered levels of fasting blood glucose and triglycerides were also found, in addition to low levels of high-density lipoprotein cholesterol. The chosen studies pointed out the frequent occurrence of cardiovascular risk factors in Military Police, with emphasis on high rates of excess body weight. In addition, the existence of relatively expressive MS prevalence data was found in the police forces, a fact that calls for special attention, requiring further studies on the subject.

Keywords: Anthropometry, Cardiovascular diseases, Obesity, Police.

1 Artigo oriundo de Trabalho Final de Graduação.

2 Nutricionista formada pela Universidade Franciscana E-mail: andressalrighi@gmail.com

3 Professora orientadora. Curso de Nutrição - Universidade Franciscana E-mail: cristiana@prof.ufn.edu.br

4 Professora do Curso de Nutrição e do Mestrado Acadêmico em Ciências da Saúde e da Vida. E-mail: natielen@ prof.ufn.edu.br 


\section{INTRODUÇÃO}

A Síndrome Metabólica (SM) é um agrupamento de distúrbios que inclui resistência à insulina, obesidade central, elevados níveis de triglicerídeos, baixos níveis de HDL e hipertensão arterial. A patologia está baseada na presença de três ou mais desses componentes em um mesmo indivíduo (FERREIRA et al., 2010).

A National Cholesterol Education Program's Adult Treatment Panel III (NCEP-ATP III definiu a SM em três ou mais critérios como: obesidade abdominal apresentando perímetro da cintura $\geq$ percentil 90 para idade e sexo; triglicerídeos elevados superior a $150 \mathrm{mg} / \mathrm{dL}$; baixos níveis de HDL-C, apresentando em homens HDL-c menor que $40 \mathrm{mg} / \mathrm{dL}$ e nas mulheres menor que $50 \mathrm{mg} / \mathrm{dL}$; elevada pressão arterial (PA), com valor igual ou superior a 135/85 mmHg, ou uso de algum medicamento para reduzir a pressão arterial; e elevada glicemia de jejum, com valor igual ou superior a $110 \mathrm{mg} / \mathrm{dL}$ ( MENDES et al., 2019).

Conforme a Associação Brasileira para o Estudo da Obesidade e da Síndrome Metabólica, o ambiente moderno é um potente estímulo para a obesidade, com aumento do consumo calórico e baixa adesão à prática de exercícios físicos. Com a crescente modernização, ocorreu um aumento significativo da prevalência da obesidade em diversas populações, inclusive do Brasil (ABESO, 2016).

A atividade exercida pelo Policial Militar (PM) é de alta periculosidade, pois são profissionais que lidam diariamente com a violência e a brutalidade. Segundo a literatura, a profissão do policial militar é uma das que mais sofre de estresse, pois trabalha sob forte tensão, muitas vezes em meio a situações que envolvem risco de vida. Além disso, pelas características da profissão, são fortes candidatos ao burnout, síndrome que se caracteriza por apresentar sintomas e sinais de exaustão física, psíquica e emocional (COSTA et al., 2007).

Um estudo realizado em Campina Grande avaliou a presença da SM em integrantes do Segundo Batalhão de Policia Militar. A amostra foi constituída de 73 profissionais, com os critérios para o diagnóstico seguindo os parâmetros do NCEP - ATP III, verificou-se 39\% dos participantes apresentavam a SM, tendo como componentes mais prevalentes a obesidade central (84,9\%), a hipertensão (75,3\%), a hipertrigliceridemia (54,7\%) e glicemia alterada (63,0\%) (CORDEIRO, 2016).

As ações diárias associadas ao trabalho do PM requerem desse profissional um ótimo desempenho, considerando que trata-se de um labor responsável por grandes desgastes físicos e psíquicos, possíveis responsáveis pelas doenças cardiovasculares (JESUS; MOTA; DE JESUS, 2014; SILVA et al., 2012).

Esse grupo laboral possui rotina diária intensa somada à ingestão calórica alta de alimentos, pouca ingestão de frutas, legumes e verduras, baixa prática de atividade física e consumo de tabaco. Todos esses fatores assim como as doenças crônicas não transmissíveis (DCNT) aumentam as chances para o surgimento da SM. No Brasil, as DCNT são consideradas um problema de saúde pública, pois são responsáveis por cerca de 72\% das causas das mortes (SILVA, 2013). 
Barbosa e Silva (2013), relatam em seu estudo que as várias situações em que o PM enfrenta acaba deixando-o vulnerável a doenças, visto que a sobrecarga do trabalho, a hierarquia rígida, dentre outros aspectos acabam expondo-o aos agravos em sua saúde.

De acordo com o artigo 144 da Constituição Federal (1988), a preservação da ordem pública é atribuição dos policiais, compreendendo o policiamento ostensivo e preventivo. Para que essas atividades sejam desenvolvidas com êxito, os militares precisam dispor de saúde física e mental. Portanto, faz-se necessário o estudo para investigar ocorrência de Síndrome Metabólica e Fatores de Risco Cardiovascular em Policiais Militares (BRASIL, 1988).

\section{MATERIAS E MÉTODOS}

A presente pesquisa é constituída de uma revisão integrativa da literatura referente à ocorrência de Síndrome Metabólica e Fatores de Risco Cardiovascular em Policiais Militares. Segundo Whittemore e Knafl (2005), o método de revisão integrativa é a única forma que consegue compilar diversas metodologias (pesquisa experimental e não experimental), tendo o potencial de delegar um papel maior na prática baseada em evidências clínicas.

O trabalho foi embasado na pesquisa de artigos realizada nos dados de Literatura Latino-Americano e do Caribe em Ciências da Saúde (LILACS), Scientific Eletronic Library Online (SCIELO) e Publicações Médicas (PUBMED). Foram utilizados os seguintes descritores do Medical Subject Headings (Mesh): Prevalence of Metabolic Syndrome, Prevalence of Cardiovascular Risk Military Police, Military Police Disease, Risco Cardiovascular em Policiais Militares, Síndrome Metabólica em Polícias Militares, Metabolic Syndrome Military Police, Fator de Risco Cardiovascular em Policiais Militares.

O procedimento de busca desenvolveu-se no mês de setembro de 2020, tendo por base artigos nas línguas portuguesa, inglesa e espanhola publicados entre 2010 e 2020, desde que disponíveis na íntegra. Para descrição dos artigos selecionados, foi elaborado um fluxograma das seleções dos estudos e uma tabela contendo autor(es); ano da publicação; base de dados originária; título; objetivos; principais resultados. Em seguida, foi realizada a discussão entre os diferentes artigos, comparando-os para, por fim, concluir os resultados alcançados após compilação dos mesmos.

Como critérios de exclusão, foram descartados: artigos publicados fora do recorte temporal estabelecido, duplicados em mais de uma base de pesquisa, teses, dissertações, artigos de revisão, protocolos, recomendações e artigos não disponíveis na íntegra.

\section{RESULTADOS E DISCUSSÕES}

Após levantamento inicial relacionado aos termos de busca citados anteriormente, foram identificados 622 artigos, sendo 285 na plataforma Pubmed, 309 na biblioteca Scielo e 28 na base Lilacs. 
A partir do refinamento, foram selecionados 11 artigos conforme demonstrado no fluxograma da figura 1. No quadro 1, são apresentados os artigos conforme os autores, ano, título, objetivo e principais resultados de cada estudo.

Figura 1 - Fluxograma da seleção dos artigos relacionados à síndrome metabólica e fatores de risco cardiovascular em policiais militares, 2020.

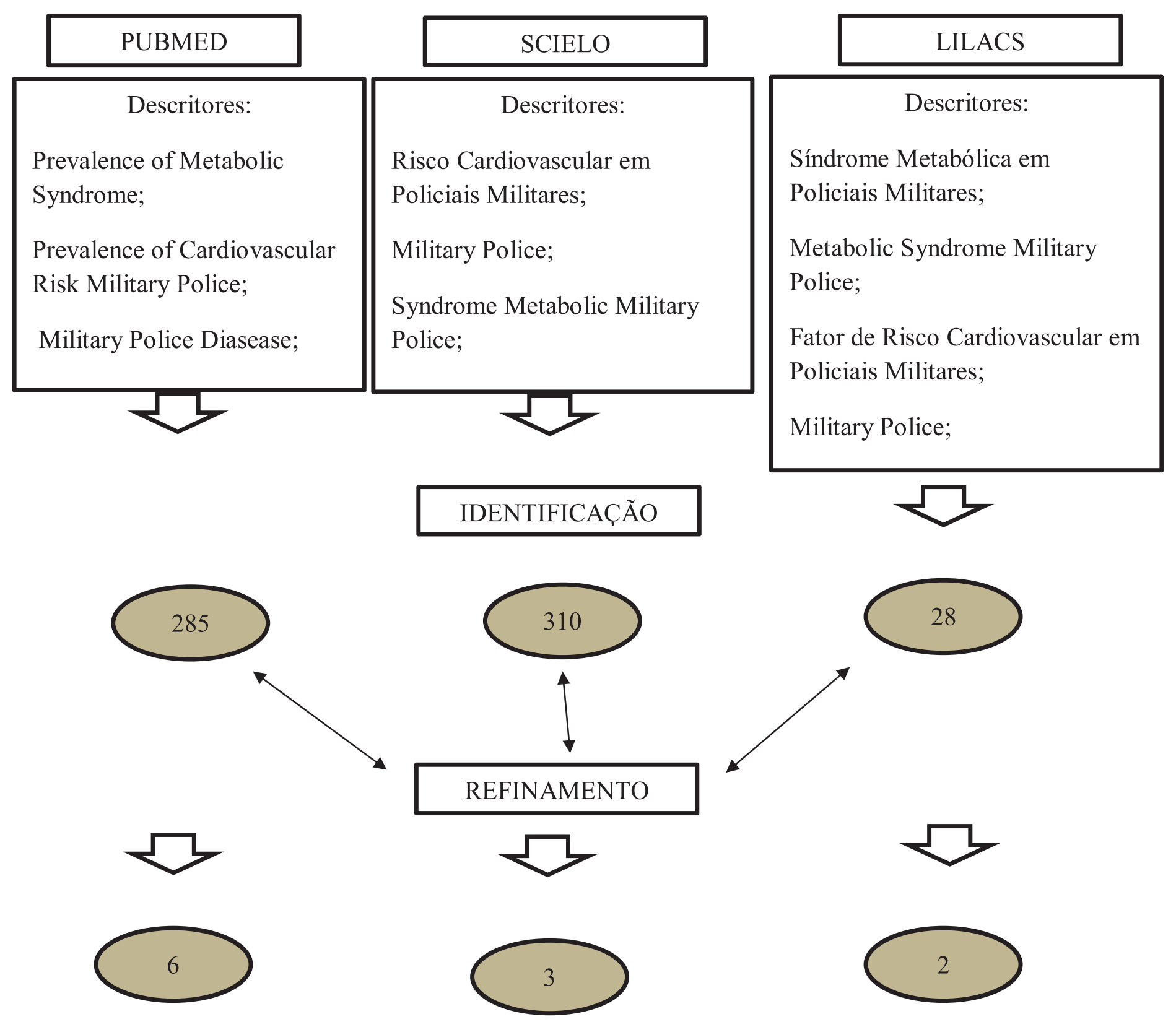

Artigos selecionados para revisão

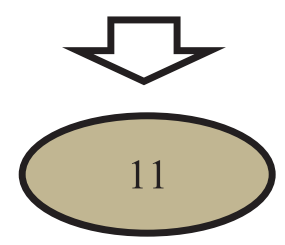

Fonte: Construção do autor. 
Quadro 1 - Artigos selecionados sobre Síndrome Metabólica e Fatores de Risco Cardiovascular em Policiais Militares, contendo autor, ano, base de dados originária, título, objetivo e principais resultados.

\begin{tabular}{|c|c|c|c|c|}
\hline Autor/Ano & Base & Título & Objetivo & Principais Resultados \\
\hline $\begin{array}{l}\text { FILHO, R. T. B.; } \\
\text { D'OLIVEIRA, } \\
\text { A., } 2013 .\end{array}$ & PubMed & $\begin{array}{l}\text { The prevalence of the } \\
\text { metabolic syndrome } \\
\text { among soldiers of the } \\
\text { Military Police of the } \\
\text { State of Bahia. }\end{array}$ & $\begin{array}{l}\text { Avaliar a prevalência de } \\
\text { fatores de risco indivi- } \\
\text { duais para doenças cardio- } \\
\text { vasculares, bem como a } \\
\text { prevalência de síndrome } \\
\text { metabólica em homens } \\
\text { que atuam no Corpo de } \\
\text { Polícia Militar do estado } \\
\text { da Bahia, Brasil. }\end{array}$ & $\begin{array}{l}\text { Os autores identificaram alta pre- } \\
\text { valência de hipertensão }(55,76 \%) \text {, } \\
\text { hipertrigliceridemia }(50,5 \%) \text {, } \\
\text { circunferência abdominal de > } \\
102 \mathrm{~cm}(31,76 \%) \text {, níveis baixos de } \\
\text { colesterol de lipoproteína de alta } \\
\text { densidade ( } 30,46 \%) \text { e glicemia } \\
\text { de jejum alterada ( } 28,15 \%) \text { A } \\
\text { prevalência geral de síndrome } \\
\text { metabólica foi de } 38,54 \% \text {. }\end{array}$ \\
\hline $\begin{array}{l}\text { SANTANA, A. } \\
\text { M. C.; GOMES, } \\
\text { J. K. V.; DE } \\
\text { MARCHI, D. } \\
\text { et al., 2012. }\end{array}$ & PubMed & $\begin{array}{l}\text { Occupational stress, } \\
\text { working condition and } \\
\text { nutritional status of } \\
\text { military police officers. }\end{array}$ & $\begin{array}{l}\text { Investigar a relação entre } \\
\text { estresse, condições de tra- } \\
\text { balho e estado nutricional } \\
\text { de } 53 \text { policiais militares } \\
\text { em uma cidade do sudeste } \\
\text { do Brasil. }\end{array}$ & $\begin{array}{l}\text { Entre os policiais estressados, } \\
73,68 \% \text { estavam com sobrepe- } \\
\text { so e obesidade, um resultado } \\
\text { significativo na medida em que } \\
\text { a obesidade pode estar rela- } \\
\text { cionada ao estresse e ambos } \\
\text { favorecem o desenvolvimento } \\
\text { de complicações metabólicas. } \\
\text { Em torno de } 31,58 \% \text { dos policias } \\
\text { militares estressados apresenta- } \\
\text { vam pressão arterial alterada. }\end{array}$ \\
\hline $\begin{array}{l}\text { DA SILVA, F. C.; } \\
\text { SALMA, S. S.; } \\
\text { VALDIVIA, B. } \\
\text { A. et al., } 2014 .\end{array}$ & PubMed & $\begin{array}{l}\text { Health-related quality } \\
\text { of life and related fac- } \\
\text { tors of military police } \\
\text { officers. }\end{array}$ & $\begin{array}{l}\text { Determinar o efeito das } \\
\text { características demográfi- } \\
\text { cas, ocupacionais, antro- } \\
\text { pométricas e dos níveis de } \\
\text { atividade física no lazer } \\
\text { sobre o risco coronariano e } \\
\text { qualidade de vida relacio- } \\
\text { nada à saúde de policiais } \\
\text { militares do estado de } \\
\text { Santa Catarina, Brasil. }\end{array}$ & $\begin{array}{l}\text { Em torno de } 83 \% \text { dos policiais } \\
\text { militares da área operacional } \\
\text { que serviram entre } 1 \text { a } 10 \text { anos } \\
\text { tinham baixos níveis de ativida- } \\
\text { de física e lazer ( } 52,1 \% \text { ) e médio } \\
\text { risco para desenvolvimento } \\
\text { doenças coronarianas }(42,4 \%) \text {. A } \\
\text { diminuição de minutos de tempo } \\
\text { de trabalho reduz um ponto a } \\
\text { mais no risco coronariano, e cada } \\
\text { diminuição da atividade física } \\
\text { levou a um ponto a mais no risco. }\end{array}$ \\
\hline $\begin{array}{l}\text { SILVA, C. A. C.; } \\
\text { LEITE, L. A.; } \\
\text { MOREIRA, A. J. } \\
\text { et al., } 2019 .\end{array}$ & PubMed & $\begin{array}{l}\text { Associação de disli- } \\
\text { pidemia, hipertensão, } \\
\text { sobrepeso/obesidade, } \\
\text { com turno e tempo de } \\
\text { trabalho entre Policiais } \\
\text { em uma pequena } \\
\text { cidade do nordeste do } \\
\text { Brasil. }\end{array}$ & $\begin{array}{l}\text { Avaliar a relação entre } \\
\text { sobrepeso/obesidade e fa- } \\
\text { tores de risco cardiovascu- } \\
\text { lar associados ao turno e } \\
\text { ao tempo de serviço entre } \\
\text { policiais militares. }\end{array}$ & $\begin{array}{l}\text { Sobrepeso/obesidade foi encon- } \\
\text { trado em } 83,3 \% \text { dos policiais. } \\
\text { Hipertrigliceridemia ( } 49,1 \%), \\
\text { baixas concentrações de } \\
\text { HDL-C (56,9\%) e LDL-C sérico } \\
\text { elevado ( } 46,1 \%) \text { apresentaram } \\
\text { associação com alterações do } \\
\text { IMC. Hipertensão arterial foi a } \\
\text { principal doença relatada pelos } \\
\text { participantes com sobrepeso/ } \\
\text { obesidade (28,2\%). No entanto, } \\
\text { alterações no IMC, dislipidemia } \\
\text { e hipertensão foram frequentes } \\
\text { entre os participantes com } 6 \text { a } 10 \\
\text { ou mais de } 10 \text { anos de serviço. }\end{array}$ \\
\hline
\end{tabular}




\begin{tabular}{|c|c|c|c|c|}
\hline $\begin{array}{l}\text { KOUWEN- } \\
\text { HOVEN- } \\
\text { PASMOOIJ, T. } \\
\text { A.; ROBROEK, } \\
\text { S. J. W.; } \\
\text { HELMHOUT, P. } \\
\text { et al., } 2015 .\end{array}$ & PubMed & $\begin{array}{l}\text { Design and baseline } \\
\text { characteristics of the } \\
\text { PerfectFit study: A } \\
\text { multicentre random- } \\
\text { ized cluster trial of a } \\
\text { lifestyle intervention } \\
\text { in employees at in- } \\
\text { creased cardiovascular } \\
\text { risk. }\end{array}$ & $\begin{array}{l}\text { Descrever o design e } \\
\text { características básicas } \\
\text { do estudo PerfectFit, que } \\
\text { está sendo conduzido por } \\
\text { funcionários com alto } \\
\text { risco cardiovascular na } \\
\text { força do trabalho militar, } \\
\text { na organização policial e } \\
\text { hospital universitário. }\end{array}$ & $\begin{array}{l}\text { Com base nessas avaliações } \\
\text { cardiovasculares, } 91,4 \% \text { foram en- } \\
\text { contrados para ter fatores de risco } \\
\text { para DCV. O risco de doenças } \\
\text { cardiovasculares na base no esco- } \\
\text { re de Framingham mostrou que } \\
\text { mais de } 30 \% \text { da população deste } \\
\text { estudo está em risco interme- } \\
\text { diário ou alto, sem diferenças na } \\
\text { distribuição entre os dois grupos. } \\
\text { Entre os participantes da força } \\
\text { policial, mais de } 76 \% \text { tinham } \\
\text { IMC maior que } 25 \mathrm{~kg} / \mathrm{m}^{2} \text {. }\end{array}$ \\
\hline $\begin{array}{l}\text { MINAYO, M. C. } \\
\text { DE S; RAQUEL, } \\
\text { G. A.; } \\
\text { OLIVEIRA, V. } \\
\text { C., } 2011 .\end{array}$ & PubMed & $\begin{array}{l}\text { O impacto das ativi- } \\
\text { dades profissionais na } \\
\text { saúde física e mental } \\
\text { dos policiais civis e } \\
\text { militares do Rio de } \\
\text { Janeiro, Brasil. }\end{array}$ & $\begin{array}{l}\text { Analisar o adoecimento } \\
\text { físico e mental de policiais } \\
\text { civis e militares do estado } \\
\text { do Rio de Janeiro, segun- } \\
\text { do condições de trabalho e } \\
\text { atividades profissionais. }\end{array}$ & $\begin{array}{l}\text { A obesidade, observada com } \\
\text { maior frequência em policiais } \\
\text { militares, constitui relevante fator } \\
\text { de risco que se associa à morte } \\
\text { por hipertensão, ao aumento do } \\
\text { colesterol e do açúcar sanguíneo. }\end{array}$ \\
\hline $\begin{array}{l}\text { BENEVIDES, } \\
\text { A.; MENDES, L. } \\
\text { R. F.; SANTIS, } \\
\text { G. C. M. et al., } \\
2018 \text {. }\end{array}$ & Scielo & $\begin{array}{l}\text { Perfil antropométrico } \\
\text { e alimentar de poli- } \\
\text { ciais militares. }\end{array}$ & $\begin{array}{l}\text { Avaliar o perfil antropo- } \\
\text { métrico e alimentar dos } \\
\text { policiais militares. }\end{array}$ & $\begin{array}{l}\text { Foi identificado que } 17 \% \text { esta- } \\
\text { vam com peso normal e } 83 \% \\
\text { com excesso de peso; } 44,7 \% \text { não } \\
\text { apresentavam risco cardiovas- } \\
\text { cular, } 36,2 \% \text { apresentam risco } \\
\text { cardiovascular e } 19,1 \% \text { com } \\
\text { risco cardiovascular aumentado. }\end{array}$ \\
\hline $\begin{array}{l}\text { DE JESUS, G. } \\
\text { M.; MOTA, N. } \\
\text { M.; DE JESUS, } \\
\text { E. F. A., } 2014 .\end{array}$ & Scielo & $\begin{array}{l}\text { Risco cardiovascular } \\
\text { em policiais militares } \\
\text { de uma cidade de } \\
\text { grande porte do nor- } \\
\text { deste do Brasil. }\end{array}$ & $\begin{array}{l}\text { Estimar a prevalência } \\
\text { e os fatores associados } \\
\text { ao risco cardiovascular } \\
\text { elevado (RCE) de policiais } \\
\text { militares, por meio do } \\
\text { indicador antropométrico } \\
\text { de obesidade abdominal. }\end{array}$ & $\begin{array}{l}\text { Em 32,3\% os PMs apresentaram } \\
\text { risco cardiovascular elevado } \\
\text { (RCE). O RCE foi mais frequen- } \\
\text { te em policiais com maior gra- } \\
\text { duação, maior tempo de polícia } \\
\text { e casados. Entre os indivíduos } \\
\text { com mais de } 10 \text { anos na polícia, } \\
\text { a taxa de prevalência de RCE } \\
\text { daqueles com graduação alta é } \\
\text { quase duas vezes maior. }\end{array}$ \\
\hline $\begin{array}{l}\text { ESTEVES, } \\
\text { J. V. D. C.; } \\
\text { ANDRADE, } \\
\text { M. L.; GEALHA, } \\
\text { L., et al., } 2014\end{array}$ & Scielo & $\begin{array}{l}\text { Caracterização da con- } \\
\text { dição física e fatores } \\
\text { de risco cardiovascular } \\
\text { de policiais militares } \\
\text { rodoviários. }\end{array}$ & $\begin{array}{l}\text { Verificar os níveis de } \\
\text { condição, composição } \\
\text { corporal e pressão arterial } \\
\text { de policiais rodoviários do } \\
\text { estado do Paraná - Brasil. }\end{array}$ & $\begin{array}{l}\text { Os policiais rodoviários do } \\
\text { presente estudo apresentaram } \\
\text { IMC classificado como obesida- } \\
\text { de leve, risco elevado e alto para } \\
\text { circunferência de cintura e RCQ } \\
\text { e percentual de gordura corporal } \\
\text { acima dos valores recomendáveis. } \\
\text { Quanto a CC foi classificada } \\
\text { como risco alto para doenças } \\
\text { cardiovasculares, extrapolando } \\
1,4 \mathrm{~cm} \text { o limite de corte. }\end{array}$ \\
\hline
\end{tabular}




\begin{tabular}{|c|c|c|c|c|}
\hline $\begin{array}{l}\text { SANTOS } \\
\text { FONTES, I. S.; } \\
\text { SAMPAIO, G. } \\
\text { C; SALTOS, S. } \\
\text { C. et.al, } 2016\end{array}$ & Lilacs & $\begin{array}{l}\text { Síndrome metabólica } \\
\text { em militares de uma } \\
\text { unidade da polícia de } \\
\text { Aracaju, Sergipe. }\end{array}$ & $\begin{array}{l}\text { Determinar a prevalência } \\
\text { de síndrome metabólica e } \\
\text { de seus componentes nos } \\
\text { militares de uma unidade } \\
\text { da Polícia em Aracaju, } \\
\text { Sergipe. }\end{array}$ & $\begin{array}{l}\text { Um total de sete ( } 7,3 \%) \text { entre os } \\
\text { participantes do estudo apre- } \\
\text { sentavam síndrome metabólica. } \\
\text { Em relação aos componentes da } \\
\text { síndrome metabólica, identifi- } \\
\text { cou-se circunferência abdominal } \\
\text { aumentada indicando risco para } \\
\text { doenças cardiovasculares em } \\
34,4 \% \text { dos participantes; sobrepe- } \\
\text { so em 57,3\%; hipertensão arterial } \\
\text { em } 11,5 \% \text {; triglicerídeos eleva- } \\
\text { dos em } 29,5 \% \text {; baixo colesterol } \\
\text { ligado a lipoproteínas de alta } \\
\text { densidade em } 60,4 \% \text {; e glicemia } \\
\text { de jejum elevada em } 31,3 \% \text {. }\end{array}$ \\
\hline $\begin{array}{l}\text { TAHAN, F.; } \\
\text { PEREIRA, J. C., } \\
2015 .\end{array}$ & Lilacs & $\begin{array}{l}\text { Avaliação do risco car- } \\
\text { diovascular por indica- } \\
\text { dores antropométricos } \\
\text { em policiais militares } \\
\text { de um batalhão do sul } \\
\text { de Minas Gerais. }\end{array}$ & $\begin{array}{l}\text { Avaliar o risco de um } \\
\text { evento cardiovascular em } \\
\text { policiais militares de um } \\
\text { batalhão do sul de Minas } \\
\text { Gerais }\end{array}$ & $\begin{array}{l}\text { O diagnóstico nutricional mais } \\
\text { frequente, independentemente } \\
\text { do tempo de serviço, em } 80 \% \\
\text { dos policiais, segundo o índice } \\
\text { de massa corporal, foi de sobre- } \\
\text { peso e obesidade, } 33 \% \text { apre- } \\
\text { sentaram risco muito elevado } \\
\text { para doenças cardiovasculares } \\
\text { associadas à obesidade pelo } \\
\text { indicador circunferência da } \\
\text { cintura e alto risco relacionado à } \\
\text { relação cintura-quadril. }\end{array}$ \\
\hline
\end{tabular}

Fonte: Construção do autor

Percebeu-se, após a análise dos artigos, que nove deles (82\%) avaliaram diretamente os fatores de riscos cardiovasculares (RCV) através de dados antropométricos, e dois (18\%) avaliaram a prevalência de Síndrome Metabólica entre os policiais militares

Em seis (55\%) desses artigos o índice de sobrepeso identificado foi de $45 \%$ a $60 \%$ além de 15\% a 20\% apresentando a condição de obesidade, ambos calculados a partir do Índice de Massa Corporal (IMC) (FONTES et al., 2016; KOUWENHOVEN-PASMOOIJ et al., 2015; MINAYO, 2011; SANTANA et al., 2012; SILVA et al., 2019; TAHAN; PEREIRA, 2015). Outrossim, dois estudos (18\%) identificaram excesso de peso variando entre $72 \%$ e $83 \%$ dos PMs, que apresentaram IMC $\geq 25 \mathrm{~kg} / \mathrm{m}^{2}$ (BENEVIDES et al., 2018; FILHO; D’OLIVEIRA, 2013).

É possível supor que essas altas taxas de excesso de peso estejam relacionadas com ritmo diário de trabalho e com a responsabilidade perante à sociedade. As situações de estresse, escalas de plantões noturnos, falta de disponibilidade de horário correto para as refeições, levam consequentemente ao ganho de peso e são fatores prejudiciais à saúde (TAHAN; PEREIRA, 2015).

Esses dados geram preocupação, visto que se espera uma boa forma dos PMs para que sejam capazes de atuar fisicamente com força e disposição suficiente para exercer suas atividades diárias durante o turno de serviço (FILHO; D'OLIVEIRA, 2013). 
Em duas cidades do nordeste, uma de pequeno porte e outra de grande porte, os PMs apresentaram respectivamente 76 e 84\% de alterações no IMC, e os que mais apresentaram risco cardiovascular elevado (RCE) foram os de graduação mais alta e com mais tempo de serviço na instituição (FILHO; D’OLIVEIRA, 2013; SILVA et al., 2019). De forma similar no sul de Minas Gerais, independentemente do tempo de serviço, cerca de $80 \%$ dos policiais encontrava-se com sobrepeso e obesidade (TAHAN; PEREIRA, 2015).

O estresse tem mostrado relação com a massa corporal dos policiais, dessa forma Santana et al. (2012) constataram que 73,8\% dos PMs estressados encontravam-se com sobrepeso ou obesidade, ratificando que tanto obesidade quanto estresse são fatores para o desenvolvimento de complicações metabólicas. Dentre os PMs com estresse, de 15 a 30\% apresentavam pressão arterial (PA) alterada, todavia os autores alertam sobre a necessidade de maiores análises para elucidação da relação entre o estresse e a alteração sanguínea (MINAYO, 2011; SANTANA et al., 2012).

Juntamente com o cálculo do IMC para a avaliação do sobrepeso e da obesidade, a medida da circunferência da cintura (CC), considerada de baixo custo, deve ser usada de maneira conjunta, a fim de se estimar a gordura corporal. Embora alguns estudos façam o uso da medida da circunferência abdominal, a medida atualmente recomendada é a da circunferência da cintura, por expor com confiabilidade o tecido adiposo visceral relacionado a doenças cardiovasculares (TAHAN; PEREIRA, 2015).

Assim, ao considerar a circunferência da cintura como parâmetro, entre 30 e $40 \%$ dos PMs estavam com risco cardiovascular muito alto (JESUS; MOTA; DE JESUS, 2014; KOUWENHOVEN-PASMOOIJ et al., 2015; TAHAN; PEREIRA, 2015). Com resultados inferiores, mas também levando em conta a circunferência, outro estudo evidenciou que $11 \%$ dos avaliados encontrava-se com RCV muito alto (SANTANA et al., 2012). Em relação a CA, de 20 a 35\% dos indivíduos apresentaram riscos cardiovasculares muito alto, sendo considerado fator coadjuvante nas complicações metabólicas associadas à obesidade (BENEVIDES et al., 2018; FILHO; D’OLIVEIRA, 2013; FONTES et al., 2016).

O sobrepeso, a obesidade, a circunferência da cintura e a relação cintura-quadril (RCQ) têm relação direta com ameaças coronarianas (TAHAN; PEREIRA, 2015). Dois estudos encontraram taxas elevadas para doenças cardiovasculares em relação à RCQ, com associação direta entre a gordura abdominal, risco de hipertensão, diabetes mellitus tipo II (DM2) e hiperlipidemias (ESTEVES et al., 2014; TAHAN; PEREIRA, 2015).

Dentre a população estudada em uma organização policial, em torno de $92,6 \%$ apresentou hiperlipidemia, um dado alarmante e um fator preocupante para RCV e SM (KOUWENHOVEN PASMOOIJ et al., 2015). Outros dois estudos mostraram que 30 a 50\% dos PM encontravam-se com triglicerídeos elevados, tendo como ponto de corte valores $\geq 150 \mathrm{mg} / \mathrm{dl}$, considerados pelo NCEP - ATP III como fator de risco para SM; e desses, 30 a 60\% apresentaram níveis baixos de colesterol de lipoproteína de alta densidade (HDL) (FILHO; D'OLIVEIRA, 2013; FONTES et al., 2016; DA SILVA, 2014). 
Entre os fatores de risco para SM, está a Diabetes Mellitus tipo 2 (DM2), 2 a 5\% de PM possuíam diagnóstico de DM2 (KOUWENHOVEN PASMOOIJ et al., 2015; SILVA et al., 2019) e 30\% dos PMs apresentavam níveis alterados de glicemia em jejum. Entretanto, os estudos em questão apresentaram resultados divergentes em relação à prevalência da SM: Nos avaliados da cidade de Salvador, a taxa de prevalência foi 38,5\%, enquanto somente 7,3\% dos PMs de Aracaju eram considerados sindrômicos (FILHO; D’OLIVEIRA, 2013; FONTES et al., 2016; SILVA et al., 2019).

Os níveis alterados de glicemia e dislipidemias estão associados a diversos fatores, um deles é o consumo alimentar. Em um estudo que avaliou essa variável, 70\% dos PM consumiam frutas apenas duas vezes na semana, 59\% só consumiam uma a duas colheres de verduras por dia, 80\% relataram ingerir refrigerante, verificando-se baixa preferência por alimentos in natura, com consequente tendência ao desenvolvimento de DCNT (BENEVIDES et al., 2018).

\section{CONCLUSÃO}

A partir de análise dos estudos relacionados à SM e aos RCV em policiais militares, foi possível evidenciar que no cenário nacional, apesar das diversas pesquisas envolvendo o grupo em questão, ainda são escassos estudos direcionados sobre a temática abordada. Conforme apurado, verificou-se correlação entre os artigos encontrados, corroborando para a assertiva de que os diversos sugestivos podem conduzir ao diagnóstico de uma síndrome metabólica.

Os estudos elegidos apontaram a frequência de fatores de risco cardiovasculares em policiais militares, com destaque para elevados índices de sobrepeso/obesidade, além de alterações bioquímicas e antropométricas. Ademais, constatou-se que há existência de dados na prevalência da SM relativamente expressivos nas forças policiais, fato que suscita a realização de novos estudos acerca do tema.

As alterações constatadas provavelmente ocorrem em virtude da falta de horários regulares para a realização das refeições e da baixa prática de atividade física, uma vez que as demandas das ocorrências acontecem em qualquer horário do dia. O horário de alimentação fixo fica prejudicado e, muitas vezes, acaba sendo realizado em locais que estão em funcionamento, onde são servidos lanches rápidos.

Como proposta para trabalhos futuros, sugere-se a inclusão de questionário frequência alimentar, visto que a variável do consumo alimentar pode estar relacionada ao excesso de peso e aos RCV constatados no público considerado. Ademais, sugere-se a inserção do gênero feminino no estudo da temática, considerando perspectiva de presença crescente das mulheres na corporação policial pode representar amplitude do conhecimento sob o prisma da população de PMs. 


\section{REFERÊNCIAS}

ABESO. Associação Brasileira para o Estudo da Obesidade e da Síndrome Metabólica. Diretrizes brasileiras de obesidade 2016. 4 ed. São Paulo. 2016.

BARBOSA, R. O.; SILVA, E. F. DA. Prevalência de fatores de risco cardiovascular em policiais militares. Revista Brasileira de Cardiologia, v. 26, n. 1, p. 45-53, 2013.

BENEVIDES, A. et al. Perfil antropométrico e alimentar de policiais militares Anthropometric profile and food behaviour of police officers. Revista Motricidade, v. 14, n. 1, p. 96-102, 2018.

BRASIL. Constituição Federal de 1988. Disponível em: https://bit.ly/3xQYXXJ

CORDEIRO, A. K. et al. Avaliação Da Síndrome Metabólica Em Policiais Militares De Campina Grande- PB. Journal of Chemical Information and Modeling, v. 53, n. 9, p. 1689-1699, 2016.

COSTA, M. et al. Estresse: diagnóstico dos policiais militares em uma cidade brasileira. Revista Panamericana de Salud Pública, v. 21, n. 4, p. 217-222, 2007.

DA SILVA, F. C. et al. Health-related quality of life and related factors of military police officers. Health and Quality of Life Outcomes, v. 12, n. 1, 2014.

ESTEVES, J. V. D. C. et al. Caracterización de la condición física y factores del riesgo cardiovascular de la policía de carreteras. Revista Andaluza de Medicina del Deporte, v. 7, n. 2, p. 66-71, 2014.

FERREIRA, A. P. et al. Predição da Síndrome Metabólica em Crianças por Indicadores. Arquivo Brasileiro de Cardiologia, p. 121-125, 2010.

FILHO, R. T. B.; D’OLIVEIRA, A. The Prevalence of Metabolic Syndrome Among Soldiers of the Military Police of Bahia State, Brazil. American Journal of Men's Health, v. 8, n. 4, p. 310-315, 2013.

FONTES, I. S. et al. Síndrome metabólica em militares de uma unidade da polícia de Aracaju, Sergipe.

Scientia Medica, v. 26, n. 3, p. 24160, 2016. 
JESUS, G. M.; MOTA, N. M.; DE JESUS, É. F. A. Risco cardiovascular em policiais militares de uma cidade de grande porte do Nordeste do Brasil. Revista Brasileira de Ciências do Esporte, v. 36, n. 3, p. 692-699, 2014.

KOUWENHOVEN-PASMOOIJ, T. A. et al. Design and baseline characteristics of the PerfectFit study: A multicenter cluster-randomized trial of a lifestyle intervention in employees with increased cardiovascular risk. BMC Public Health, v. 15, n. 1, p. 1-14, 2015.

MENDES, M. G. et al. Prevalence of Metabolic Syndrome ad associations with nutrional status in adolescentes, Caderno Saúde Coletiva, 2019.

MINAYO, M. C. DE S.; DE ASSIS, S. G.; DE OLIVEIRA, R. V. C. Impacto das atividades profissionais na saúde física e mental dos policiais civis e militares do Rio de Janeiro (RJ, Brasil). Ciência e Saúde Coletiva, v. 16, n. 4, p. 2199-2209, 2011.

SANTANA, Â. M. C. et al. Ocuppational stress, working condition and nutrional status of military police officers. Work, v. 41, n. SUPPL.1, p. 2908-2914, 2012.

SILVA, J. M. DA C. Et al. Prevalência e Fatores Associados à Síndrome Metabólica em Militares do Exército Brasileiro. Dissertação de mestrado, 2013.

SILVA, C. A. C. et al. Association of dyslipidemia, hypertension and overweight/obesity with work shift and duration of employment among police officers in a small town in Northeastern Brazil. Revista Brasileira de Medicina do Trabalho, v. 17, n. 4, p. 537-544, 2019.

SILVA, R. et al. Aspectos relacionados à qualidade de vida e atividade física de policiais militares de Santa Catarina - Brasil. Motricidade, v. 8, n. 3, p. 81-89, 2012.

TAHAN, F. PEREIRA, J. C. Avaliação de risco cardiovascular por indicadores antropométricos em policiais militares de um batalhão do Sul de Minas Gerais Assessment of cardiovascular risk by anthropometric indicators in military policemen of a battalion in the South of Minas Gerais. Nutrição Brasil, v. 84, 2015.

WHITTEMORE, R; KANAFL, K. The integrative review: updated methodology. Methodological issues in nursing research, 2005. 
